Revue d'histoire de l'Amérique française

RAS REVUE D.HISTOIRE DE L'AMÉRIQUE FRANÇAISE

\title{
Histoire, neutralité et Autochtones : une longue histoire...
}

\section{Pierre Trudel}

Volume 53, numéro 4, printemps 2000

Histoire des Premières Nations : nouvelles lectures et nouveaux

problèmes

URI : https://id.erudit.org/iderudit/005626ar

DOI : https://doi.org/10.7202/005626ar

Aller au sommaire du numéro

\section{Éditeur(s)}

Institut d'histoire de l'Amérique française

\section{ISSN}

0035-2357 (imprimé)

1492-1383 (numérique)

Découvrir la revue

\section{Citer cet article}

Trudel, P. (2000). Histoire, neutralité et Autochtones : une longue histoire.... Revue d'histoire de l'Amérique française, 53(4), 528-540.

https://doi.org/10.7202/005626ar d'utilisation que vous pouvez consulter en ligne.

https://apropos.erudit.org/fr/usagers/politique-dutilisation/ 


\section{$\mathrm{H}$ istoire, neutralité et Autochtones: une longue histoire...}

PIERRE TRUDEL

Département d'anthropologie

Cégep du Vieux Montréal

'HISTOIRE DOIT-ELLE rester neutre à propos des Autochtones, se limiter à fournir strictement de l'information et éviter de jouer un rôle politique comme par exemple, nourrir la conscience identitaire des Premières $\mathrm{N}$ ations ou justifier leurs revendications territoriales; doit-elle, au contraire, faciliter l'intégration de ces dernières dans la société nord-américaine? J'avoue être un peu étonné par la façon de poser le problème; pour comprendre ma réaction, reprenons la formulation de la question posée par la Revue d'histoire de l'Amérique française L'histoire doit-elle rester neutre à propos des Québécois, se limiter à fournir strictement de l'information et éviter de jouer un rôle politique, comme par exemple, nourrir la conscience identitaire des Québécois, justifier la notion d'intégrité territoriale du Québec ou la loi 101; doit-elle, au contraire, faciliter l'intégration des Québécois à la société nordaméricaine?

II me semble que la façon de formuler le problème par la RHAF montre une crainte plus grande de sombrer dans la politique lorsqu'il est question des Autochtones. Sylvie Vincent faisait remarquer qu'un des effets du débat suscité par la panution de son livre, au tournant des années 1980, fut celui-ci : de peur d'être accusés de racisme, les historiens avaient désormais tendance à éviter la question autochtone; ils ne savaient plus comment en parler. Est-on toujours sous le choc de L'image 
de l'Amérindien dans les manuels scolaires du Québec ou Comment les Québécois ne sont pas des sauvages ${ }^{1}$ ?

L'histoire n'est jamais neutre et la question posée par la RHAF m'amène plutôt à me poser celle-ci: doit-il y avoir une «histoire nationale» des Premières Nations? Sans cet élément de référence, les historiens sont-ils condamnés à perpétuellement s'éloigner d'une perspective plus juste de la place des Autochtones dans I'histoire? La neutralité découle d'efforts d'objectivité qu'on peut évaluer isolément. Cependant, la démarche scientifique se réalise dans des contextes plus vastes. J'aborderai ici comment ces contextes limitent nos prétentions à la neutralité. Connaître ou ne pas connaître des versions autochtones de I'histoire détermine grandement le contexte dans lequel s'écrit la reconstruction du passé. N otons que je n'ai pas effectué une analyse exhaustive de la place actuelle des Autochtones dans l'historiographie et les manuels scolaires, je me suis limité à consulter quelques auteurs. Comme le souhaitent les organisateurs de cette «table ronde écrite», je livre une réflexion libre qui soulève des questions, favorise le débat et lance des pistes de recherche.

Ia rencontre des histoires nationales

Qu'est-ce que I'histoire nationale? Gérard Bouchard répond à cette question en écrivant qu'il s'agit d'un «genre scientifique» qui renvoie à la question de l'identité. «Dès le départ, l'histoire nationale ainsi définie institue un nous qu'elle raconte et à qui elle s'adresse et un autre qui n'intervient jamais comme sujet? ${ }^{2}$. Ce genre scientifique pose bien entendu des problèmes d'objectivité. On le retrouve plus particulièrement dans les programmes scolaires où il devient en quelque sorte l'histoire officielle, «prise en charge par l'État ${ }^{3} »$. Selon Bouchard, les Québécois devraient avoir une histoire nationale; il propose une série de principes sur lesquels elle devrait s'appuyer. Par exemple, cette histoire devrait inclure les diverses composantes ethniques ou minoritaires; elle devrait être sociale, c'est-à-dire ne pas axer son récit strictement sur le développement de l'État. Bien que s'adressant à un nous, tout en participant bien sûr à son développement, I'histoire nationale devrait être comparative: «il s'agit de confronter son évolution et ses singularités à

1. Cahiers du Q uébec (M ontréal, H urtubise HMH, 1979), 326 p.

2. Gérard Bouchard, La nation québécoise au futur et au passé (Montréal, VLB éditeur, coll. «Balises», 1999), 87.

3. Ibid., 89. 
des évolutions et à des expériences collectives qui ont déjà été vécues par d'autres populations, et souvent en parallèle $e^{4}$.»

Devrait-il y avoir également une «histoire nationale des Autochtones», distincte de celle proposée par Bouchard? Et de laquelle découlerait une place plus juste de ces demiers dans I'histoire générale et les manuels scolaires? À mon avis, pour les mêmes raisons que s'élaborent au fil du temps diverses histoires nationales ou versions de l'histoire, la situation politique contemporaine fait en sorte que s'écrit présentement une histoire nationale des Autochtones du Canada. On n'a qu'à jeter un coup d'œil sur le volumineux rapport de la Commission royale d'enquête sur les peuples autochtones pour constater la tendance à la rédaction d'une telle histoire officielle. Nettement, on passe d'une tradition orale locale ou plutôt, on ajoute à celle-ci une histoire écrite qui participe à la construction de l'identité de l'Autochtone canadien. Ainsi, doit-on réajuster «la nôtre» en tenant compte de ce récit historique qui ne dissimule nullement le fait qu'il ait pour fonction de légitimer des revendications politiques? Afin de ne pas percevoir la paille strictement dans I'œil du voisin, il s'avère nécessaire d'examiner brièvement la place des Autochtones dans nos manuels scolaires et notre historiographie.

I es a tochtones dans l'histoire: un portrait flou

Jean-Paul Bernard" ${ }^{5}$, dans son article "L'historiographie canadienne récente (1964-1994) et I'histoire des peuples au Canada», constate que de profonds changements ont caractérisé les historiographies canadienne et québécoise et vont dans le sens de l'amélioration, bien que certains problèmes demeurent. Pour ce qui est des Autochtones, l'auteur s'appuie sur l'étude de James R. Miller (1994) et écrit qu'après avoir été dans un premier temps quasi absents pour ensuite devenir de simples éléments du décor de l'histoire du Canada à ses débuts, ils sont devenus de nos jours de véritables acteurs importants ${ }^{6}$. Ainsi, selon Miller et d'autres auteurs, dans la dernière décennie, «on [aurait] fait plus et mieux, à la fois du côté de l'histoire des relations avec les Autochtones et du côté de I'histoire des Autochtones eux-mêmes (Indiens Inuit et Métis). Aujourd'hui cette historiographie échapperait mieux qu'autrefois à la simplification outrancière qui conduit à considérer a priori, dans

\footnotetext{
4. Ibid., 101.

5. The Canadian Historical Review, 76,3 (septembre 1995).

6. Ibid., 349.
} 
toutes les situations, les communautés autochtones comme des victimes, elle serait plus ouverte à l'utilisation des sources autochtones, et elle ne demanderait pas mieux que la participation de chercheurs autochtones à son entreprise ${ }^{7} »$. Et l'auteur d'ajouter que, comme pour ce qui est des études sur l'immigration et les groupes ethniques, «c'est là où les Autochtones ont la plus grande importance relative dans la population (Prairies, Colombie-Britannique et Nord correspondant) que les études sur cette population d'origne ancienne sont les plus développées. C'est là aussi où ces études réussissent le mieux à intéger cette histoire à ce qu'à un certain point on peut appeler I'histoire générale. Dans le cas de l'O ntario et du Québec on connaît mieux les Autochtones de leur partie nord ${ }^{8} \gg$.

Bouchard, quant à lui, n'écrit pas que les Autochtones derraient avoir leur histoire nationale; cependant, I'histoire nationale québécoise doit opérer de profonds changements afin de tenir compte correctement des Autochtones. II remet en question le mythe des fondateurs européens: «Cette représentation des origines en dit long sur la distance culturelle et politique qui sépare actuellement les Québécois francophones et les Amérindiens. » II suggère que l'on commence le récit du peuplement du territoire par l'immigration amérindienne en Amérique du Nord. De plus: "Dans I'historiographie québécoise, contrairement à celles des États-Unis, de la Nouvelle-Zélande et de l'Australie, ici on chercherait vainement une véritable tradition critique à l'égard des Européens ou des Blancs en général et adoptant le point de vue des Autochtones: une tradition qui, systématiquement, installerait les immigrants européens dans le rôle d'envahisseurs ${ }^{10} \ldots$.. Bouchard souhaite que le regard sur les réalités amérindiennes se transforme et $s^{\prime}$ affine ${ }^{11}$. II relève quelques énoncés étonnants, retrouvés au hasard de ses lectures, et qui démontrent le contraire d'une grande sensibilité. L'unique indication quantitative qu'il nous présente est celle-ci: dans les trente dernières années, la RHAF n'a consacré que $1 \%$ de ses textes aux Amérindiens.

J'ai consulté deux textes sur la place des Autochtones dans les manuels scolaires. Les deux auteurs semblent arriver à des conclusions différentes. L'un affirme que depuis L'image de l'Amérindien dans les manuels

7. Ibid.

8. Ibid., 349-350.

9. G. Bouchard, op. cit., 118.

10. Ibid., 116.

11. Ibid., 134. 
scolaires du Québec de Arcand et Vincent (1979), la situation a bien changé; l'autre pense plutôt le contraire. Comparons leurs conclusions:

On voit bien comment la description actuelle de l'Amérindien tranche avec la précédente. Celui-ci a statut d'acteur majeur, avant l'arrivée des Européens tout autant qu'aux premiers temps de la colonie. II joue un rôle important dans le succès de l'enracinement français en terre américaine. Sous certain angle, il apparait comme la victime de l'expansion européenne: on sent là l'expression d'une culpabilité blanche exprimant bien la rectitude politique de notre époque. Au fur et à mesure que la colonie se développe, I'Amérindien disparaît toutefois du décor pour ne réapparaitre que dans les années 1980, au moment des grandes confrontations entre autochtones et blancs pour ce qui est des droits territoriaux. En fait, bien que l'on reconnaisse volontiers que l'Amérindien appartiendra à l'histoire du territoire américain, il reste en marge de celle de la civilisation qui s'y installe et qui, dans son expansion, l'engloutit ${ }^{12}$.

Christian Laville, pour sa part, affirme que: «M ême dans leur dernière mouture, les manuels restent fortement marqués par cet ethnocentrisme qu'avaient observé Sylvie Vincent et Bernard Arcand. Les Blancs qui font les manuels font I'histoire des Blancs et ramènent tout à eux. Même s'ils prétendent, à l'occasion, faire l'histoire de tous ${ }^{13}$.» «En fait, on garde l'impression que ce que Vincent et Arcand avaient observé dans les manuels des années 1970 perdure dans ceux qui les ont remplacés: les Amérindiens sont là lorsqu'ils servent de référence à l'histoire des Blancs, lorsqu'ils contribuent, finalement, à justifier l'identité spécifique des exFrançais (Canadien français, Q uébécois) ${ }^{14}$.»

Le portrait actuel de la représentation des Amérindiens dans les manuels scolaires et dans I'historiographie n'est pas très clair. Les questions posées par la RHAF en témoignent sûrement, d'où la nécessité de mener des études dans ce domaine. Sans doute que les conclusions des deux analystes de nos manuels scolaires, à savoir si cette historiographie s'éloigne ou pas de celle analysée par Vincent et Arcand, ne sont pas si éloignées, car être «engloutie par la civilisation» ou servir «de référence» dans le cadre de la construction de l'identité des Blancs n'est pas très différent. Les analyses de Bouchard et de Bernard soulèvent la

12. Jocelyn Létourneau, «Nous autres les Québécois», International Textbook Research, 18 (1996): 281.

13. Christian Laville, «Les Amérindiens d'hier dans les manuels d'histoire d'aujourd'hui », Traces, 29,2 (1991): 26.

14. Ibid., 28. 
question d'une représentation différente de l'Autochtone dans I'histoire du Québec et du Canada. D'autre part, s'il s'avère que I'histoire du Québec en général, comme les manuels scolaires, ne participent pas au développement de l'identité des Amérindiens comment ceux-ci pourraient-ils s'associer à une entreprise qui vise la construction de l'identité de l'Autre plutôt que la leur? À moins qu'ils décident de devenir plutôt Québécois que Canadiens ce qui ne m'apparaît pas être le cas présentement. Cette situation explique sûrement, à mon avis, le malaise observé par Bouchard face au rapport entre les historiens et la question des Autochtones ${ }^{15}$.

\section{il n'y a pas de vide dans la nature... de l'histoire}

II semble donc que le problème actuel quant à la situation des Autochtones dans I'historiographie, plus particulièrement québécoise, soit double: un problème de perspective ou, comme l'écrit Bouchard, un manque de sensibilité, mais encore, et surtout, l'absence des Autochtones dans la période de la formation de l'État canadien (ils reviennent soudainement dans les années 1980 sous la frme de problèmes politiques). Ce n'est pas un détail puisque, dans le contexte actuel, ce vide sert à la nouvelle forme de négation de leur identité. Ainsi, il n'est pas étonnant de constater que le volume 1 du rapport de la Commission royale d'enquête sur les peuples autochtones vise précisément à contribuer à une meilleure perspective, mais ainsi à combler le vide dont il est question plus haut. Financée par l'État, jumelant une approche canadienne et autochtone de faire I'histoire, I'«histoire officielle» de la Commission royale, commission composée pour moitié d'Autochtones, présente un récit qui consolide une identité en créant littéalement un nous autochtone canadien, tout en répliquant à la forme moderne de négation de cette identité. Autrement dit, en prenant comme point de comparaison les anciens manuels scolaires ou l'historiographie ancienne, la place des Autochtones dans I'historiographie et dans les manuels scolaires actuels semble s'améliorer, mais si on la compare avec le récit historique de la Commission royale, on a plutôt l'impression que les historiens et les auteurs de manuels actuels s'adonnent toujours à la négation de l'Autre.

15. Lors d'un débat organisé par l'Association québécoise d'histoire politique (17 septembre 1999), Gérard Bouchard affirma qu'il ne voyait pas comment résoudre le problème de I'intégration des Autochtones à I'histoire du Québec et ce, après avoir réaffirmé, avec plus d'insistance que dans son livre, que leur place actuelle dans notre histoire posait de graves problèmes. Pour le moment, selon lui, nous en sommes à l'étape de débattre de la question. 
Ce que j'entends ici par négation de l'Autre consiste à faire abstraction, à minimiser, à caricaturer des faits qui sont centraux dans l'argumentation de l'Autre. L'histoire des Autochtones, ou la place de ceux-ci dans l'histoire en général, devrait consolider leur identité. L'État canadien a mené jusqu'à récemment des politiques qui visaient à réduie grandement l'identité autochtone. Faire abstraction de ces politiques dans l'élaboration du récit historique, y attribuer peu d'importance ou les présenter trop hâtivement, ce qui leur enlève toute signification, constituent à mon avis une forme moderne de négation de l'Autre. L'argument selon lequel l'État du Québec riest pas directement responsable de ces politiques sert avant tout le processus de définition de l'identité québécoise. Pour les Autochtones, il s'agit de la négation de leur argument principal.

Voyons maintenant plus précisément comment joue cette nouvelle forme de négation ${ }^{16}$. Dans les 600 pages du volume 1 du rapport de la Commission royale d'enquête, quelque 50 pages sont consacrées respectivement à chacune des étapes de l'histoire des Autochtones du Canada. Par la suite, le récit revient sur la troisième étape, soit celle qui est plus particulièrement absente dans notre récit historique. Plus de 400 pages expliquent alors en détail comment l'État canadien a tenté d'assimiler les Autochtones et de détruire, à long terme, leur identité ! Le mandat de la Commission consistait, entre autres, à examiner les relations historiques entre l'État canadien et les Autochtones, ce qui explique probablement en partie la présence de ces 400 pages; mais également, celles-ci visent sûrement à combler le vide de notre histoire nationale. La périodisation de l'histoire nationale autochtone de la Commission comporte quatre étapes: 1 - Deux mondes étanches; 2 - Interaction et coopération; 3 - Déracinement et assimilation; 4 - Négociation et renouveau. La Commission propose de retourner au type de relation qui caractérise les nations entre elles et ce type de relation politique occupe la plus vaste période de I'histoire depuis l'arrivée des Européens. II s'agit de la seconde étape. Le message est très clair. Rien de plus facile, de moins étrange et de plus rassurant, que de retourner à ce qui a caractérisé la majeure partie de l'histoire du Canada ! Puisque les diverses histoires nationales se répondent entre elles, il reste maintenant à vérifier si,

16. J'ai montré ailleurs comment en général dans la société s'exprime la négation de l'Autre, voir: «De la négation de l'Autre dans les discours nationalistes des Québécois et des Autochtones», Recherches amérindiennes au Québec, 25,4 (1995-1996): 53-66. 
depuis que les Autochtones sont de retour sous la forme d'un problème politique, nous n'aboutissons pas, à la lecture de notre histoire nationale, à recevoir exactement le message contraire. Autrement dit, à nous poser cette question: W hat do the Indians want? La différence de perspective que l'on pourrait constater serait celle-ci : d'un côté, rien de plus facile que de retourner à ce qui a presque toujours existé, tandis que de l'autre, on n'arrive pas trop à comprendre comment tenir compte de la présence actuelle d'Autochtones, eux qui ont été littéralement absents de l'histoire du pays. Comment donc concilier avec la modernité ces étranges «droits ancestraux» quasiment préhistoriques puisqu'ils n'existent pas dans l'histoire de la formation de l'État?

II n'y a pas que le nombre de pages accordées à telle ou telle autre question qui différencie nos «histoires officielles», il y a aussi le niveau de précision dans l'information. Rien de plus naturel de nos jours que d'apprendre, en lisant un texte historique, que des Autochtones ont perdu des terres. Mais quelles terres? Quand? Comment? Depuis 150 ans, les Autochtones ont perdu $60 \%$ des terres qui leur avaient été réservées, apprend-on à la lecture du rapport de la Commission. Ces terres se trouvent la plupart du temps autour des réserves actuelles. Ainsi, on peut se poser cette question: pourquoi cette information n'existe-t-elle pas dans notre histoire? Existe-t-il une carte géographique, dans un texte historique ou dans un manuel scolaire, de la localisation de ces réserves et du changement dans leurs superficies au cours du dernier siècle, un peu comme des manuels illustrent les modifications de la superficie des provinces? Quant aux nébuleux «droits ancestraux», expliqués longuement dans le texte de la Commission - surtout comment ils ont survécu jusqu'à maintenant et comment ils se défnissent actuellement au fil des ententes politiques et des interventions de la Cour suprême - , est-il exact que, même encore aujourd'hui, aucun texte d'histoire générale n'informe du fait que le tiers des dispositions de la Proclamation royale concerne ces fameux droits ancestraux, et du fait que des dispositions stipulent les modalités d'acquisition du teritoire indien? Retrouve-t-on dans des textes d'historiens ou d'histoire générale des références à des extraits de jugement de la Cour suprême contribuant à la définition plus précise de ces droits dans un contexte moderne?

À la suite de la crise de Listuguj en Gaspésie, quant aux activités forestières jugées illégales par le Québec, une enquête Sondagem-Le Devoir (25 août 1998) indique «un vif sentiment de ras-le-bol » dans la 
population à l'égard des revendications des Autochtones: 70,5\% des Québécois sont convaincus que «les manifestations des Autochtones visent d'abord et avant tout à faire chanter le gouvernement pour lui soutirer de l'argent». Cinquante-quatre pour cent (54\%) des Québécois veulent que l'armée intervienne si des Autochtones bloquent une route. En collaboration avec un journaliste autochtone de Listuguj, j'ai montré comment les médias ont littéralement occulté la question des droits ancestraux ${ }^{17}$. II est un peu hallucinant de mesurer la distance séparant d'un côté des historiens qui craignent de sombrer dans la politique en abordant les questions autochtones et, de l'autre, la population qui demande l'intervention de l'armée parce que les Autochtones tentent de soutirer de l'argent au gouvernement !

Je ne propose pas ici que les Autochtones tiennent une place démesurée dans l'histoire. Mais de quoi parle-t-on? De quelle façon et avec quelles précisions? À mon avis, tenir compte des versions autochtones de l'histoire qui nous sont disponibles favorise sûrement une histoire plus neutre. Bien entendu, les sensibilités ne seront jamais les mêmes on n'a qu'à penser à I'histoire du Canada et du Québec -, mais l'absence chronique des Autochtones dans la période de la formation de l'État accentue le problème de distorsion quant à l'interprétation du passé. Et cette absence mène clairement à rendre incompréhensibles les enjeux contemporains, d'où la facilité qu'ont les journalistes à les occulter. Pourquoi les Autochtones s'associeraient-ils à une telle histoire?

pl us d'historiens que d'indiens...

Dans son article, Jean-Paul Bernard écrit que la montée fulgurante des effectifs, soit le nombre grandissant d'étudiants et de professeurs d'histoire, constitue un facteur qui a contribué au développement d'une nouvelle histoire plus diversifiée et qui montre l'élargissement de ses préoccupations ${ }^{18}$. Les données récentes de Statistiques Canada indiquent que ceux qui lisent et qui font I'histoire sont rarement autochtones (Même le récit de la Commission royale d'enquête a été largement écrit par des non-Autochtones, ce qui, dans le contexte actuel, ne lui enlève rien de sa pertinence.) Bien qu'il faille considérer l'élargissement de la définition d'un Autochtone, Statistiques Canada indique que le nombe

17. Pierre Trudel et Félix Atencio-Gonzalez, «Les médias et les Micmacs - H uit ans après la crise d'Oka, les journalistes québécois comprennent-ils mieux le dossier autochtone?», Le Trente (novembre 1998) : 31-34.

18. J.-P. Bernard, loc. cit., 334. 
d'entre eux qui ont obtenu un diplôme d'études supérieures (collège et université) a doublé entre 1986 et 1996; cependant, $3 \%$ des étudiants ont terminé des études universitaires, comparativement à $15 \%$ chez les nonAutochtones ${ }^{19}$. Cette situation nous oblige à une certaine prudence dans l'évaluation de la place des Autochtones dans l'histoire et dans l'évaluation de ce qui, à une époque donnée, nous apparaît constituer la «neutralité». Elle devrait nous amener à être très sensible, et curieux, à l'égard des diverses perspectives autochtones de l'histoire. Bien que l'effectif d'historiens autochtones soit faible, on ne peut faire autrement que d'attribuer beaucoup d'importance à ce qui en quelque sorte constitue actuellement un indice de ce qui n'est pas encore sur papier et qui, un jour nous l'espérons, deviendra un outil permettant d'ajuster nos sensibilités et nos perspectives.

l'autre côté de la médaille:

les a bus a mérindiens de la mémoire

«Go back to Europe» est probablement l'une des demières phrases que le caporal Lemay a entendues le matin du 11 juillet 1990 à Oka, selon des témoins à l'enquête du coroner Gilbert sur la mort de ce policier. Les abus de la mémoire est le titre d'un petit livre de Tzevetan Todorov dans lequel l'auteur explique ce qui peut aussi contribuer à une meilleure perspective de I'histoire des Autochtones. II aborde la délicate question de groupes opprimés qui s'adonnent aux abus de la mémoie en exagérant leur statut de victimes dans le but, entre autres, d'en tirer profit, profit qui n'est pas nécessairement matériel. La victime se complaît dans l'établissement d'une «dette symbolique»; «[...] cela lui ouvre dans le présent une ligne de crédit inépuisable»; «[...], or, plus grande a été l'offense dans le passé, plus grands seront les droits dans le présent ${ }^{20} »$. Et bien entendu, ces droits iront parfois jusqu'à celui de tirer sur des policiers...

L'auteur établit deux types de mémoire: la mémoire littérale et la mémoire exemplaire. "L'usage littéral, qui rend l'événement ancien indépassable, revient en fin de compte à soumettre le présent au passé. L'usage exemplaire, en revanche, permet d'utiliser le passé en vue du présent, de se servir des leçons des injustices subies pour combattre celles qui ont cours aujourd'hui, de quitter le soi pour aller vers

19. Heather Tait, «Niveau de scolarité des jeunes adultes autochtones», Tendances sociales canadiennes (printemps 1996): 7.

20. Les abus de la mémoire (Paris, Arléa, 1998), 56. 
I'autre ${ }^{21}$. » Todorov relate comment il s'est senti mal à l'aise en participant à un congrès au musée d'Auschwitz qui portait sur «L'unicité et I'incomparabilité de I'holocauste». II rappelle également que l'état d'esprit créé par une mémoire trop littérale a mené des Israéliens et des Palestiniens à déclarer, à propos des discussions sur Jérusalem, que «pour simplement commencer à parler, il faut mettre le passé entre parenthèses ${ }^{22} »$. Dès le départ, l'auteur établit que pour les peuples ou les individus opprimés, la mémoire, comme un instinct de survie, s'oppose à I'histoire officielle qui les ignore. Elle s'oppose par exemple aux bourreaux nazis dans leurs efforts de ne laisser aucune trace: «La vie a perdu contre la mort, mais la mémoire gagne dans son combat contre le néant ${ }^{23}$. Cependant, il y a aussi les abus de la mémoire; «[... ] cette foisci, le passé sert à refouler le présent, et ce refoulement n'est pas moins dangereux que l'autre. Tous ont le droit de recouvrer leur passé, certes, mais il n'y a pas lieu d'ériger un culte de la mémoire pour la mémoire; sacraliser la mémoire est une autre manière de la rendre stérile ${ }^{24}$. »

Bien que, dans notre histoire, nous puissions en quelque sorte compter les morts politiques sur les doigts de nos mains comparativement aux situations traitées par Todorov, il n'en reste pas moins que les principes généraux qu'il dégage s'y appliquent également. On tente sûrement, ici aussi, présentement, d'échapper à une mémoie trop littérale. Bernard écrit à propos de l'historiographie québécoise actuelle: «Elle tend ainsi à dédramatiser, voire à mettre entre parenthèses, les difficultés ou les malheurs évoqués plus traditionnellement dans I'historiographie antérieure du Canada français $s^{25}$. $\gg$ Ce qui mènerait, selon lui, au débat actuel : "D'où la tendance dans la "nouvelle" histoire du Québec à gommer ce que l'héritage de la conquête a pu impliquer de conflictuel dans les relations entre la majorité francophone et la minorité anglophone ${ }^{26} \ldots{ }^{\prime \prime}$

On comprend facilement que les 600 pages du récit de la Commission royale d'enquête sur les peuples autochtones ne gomment rullement les effets du colonial isme canadien ! Au contraire. II s'agit substantiellement

\author{
21. Ibid., 31. \\ 22. Ibid., 26. \\ 23. Ibid., 16. \\ 24. Ibid., 33. \\ 25. J.-P. Bernard, loc. cit., 341. \\ 26 Ibid., 342.
}


de quatre chapitres qui traitent des pensionnats de la Loi sur les Indiens, de la réinstallation de collectivités autochtones et du sort injuste réservé aux anciens combattants des Premières $N$ ations. II serait sûrement plus facile d'identifier les abus de la mémoire de ce côté-là si les Autochtones étaient mieux représentés dans notre historiographie et nos manuels scolaires, ce qui réduirait sûrement la crainte des historiens d'être accusés de «racisme» lorsqu'ils dénoncent ces abus. Par ailleurs, selon le modèle proposé par Todorov et par Bouchard, I'histoire des Autochtones devrait aussi être comparative ${ }^{27}$. Les auteurs du récit de la Commission ont-ils suffisamment fait appel à cet exercice de comparaison? Ont-ils eu plutôt tendance à créer une victime au caractère d' «unicité et d'incomparabilité»?

Dans son commentaire, publié par Recherches amérindiennes au Q uébec ${ }^{28}$, Trigger fait remarquer que le récit historique de la Commission ne compare jamais la situation des Autochtones canadiens avec celle, toute proche, des Amérindiens des États-Unis Vincent et Dickason, quant à elles, relèvent le fait que rien n'est dit de la période de la Nouvelle-France, comme si elle n'existait pas. Dickason ajoute que si la Commission fait un effort de relativisme en rappelant que les responsables du malheur des Autochtones, soit les Européens, n'ont pas été les seuls coupables à avoir bâti des empires aux dépens d'autres populations. Ces efforts ne sont cependant pas suffisants, selon I'historienne autochtone, puisque la Commission ne précise pas que l'un de ces plus vastes empires a été «de fabrication amérindienne». J'ajouterai que le récit de la Commission fait en sorte que le lecteur n'est jamais amené à comparer la situation des Canadiens français et des Québécois avec celle des Autochtones, malgré le fait qu'il s'agisse manifestement d'un problème politique de même nature, et surtout des pratiques d'un même État. C'est comme si on voulait empêcher une association d'idées qui ne favoriserait pas le développement d'une certaine identité nationale autochtone... Dans le processus de définition des identités, peut-être

27. Non pas du genre de cette comparaison que l'on trouve dans un éditorial d'un journal amérindien (Eastern Door, journal mohawk) qui établit littéralement une équation entre I'holocauste et la situation des Autochtones canadiens, et ce, en rappelant les grandes lignes de ce que contient le rapport de la Commission. J'ai eu l'occasion de lire des versions non définitives de ce rapport qui contenaient l'expression «génocide»; heureusement, on ne la trouvait plus dans la version finale.

28. Voir «Dossier spécial sur le rapport de la Commission royale sur les peuples autochtones», Recherches amérindiennes au Québec, 27,3-4 (hiver 1997): 107-129. 
commence-t-on par bien marquer les événements passés au moyen d'une mémoire davantage littérale, pour ensuite passer à une mémoire exemplaire qui permet d'établir des comparaisons et de relativiser les situations? L'histoire n'est pas au service du processus de définition des identités, mais ces processus produisent des contextes qui établissent des normes, et des perspectives générales, et celles-ci influencent inévitablement les historiens

Comme pour toute histoire, il est sûrement difficile de se limiter à fournir strictement de l'information et à ne pas jouer un rôle politique. Le débat devrait porter sur comment tenir compte de ces diverses histoires nationales, et puisque la tendance générale nous y amène, à évaluer aussi dans quelle mesure les Autochtones ont été des victimes Dans ce débat, il ne faudra pas s'étonner de retrouver des abus «amérindiens» de la mémoire, tout comme il y a eu, et qu'il y a encore, une absence quasi totale des Amérindiens dans notre histoire, plus particulièrement à partir du $\mathrm{xviI}^{\mathrm{e}}$ siècle jusque dans les années 1980. La plus grande sensibilité proposée par Bouchard pourrait ainsi rencontrer sur son passage l'ouvrage d'un historien amérindien, intitulé Red Earth W hites Lies - Native American and the M yth of the Scientifique Fact ${ }^{29}$, dans lequel l'auteur, très alerte dans la défense du statut de premier habitant, tente de démontrer en long et en large qu'il n'y a pas plus de preuves qui confirment le peuplement de l'Amérique par l'Asie que le contraire. 